\title{
Relationship for Quotient Moments of Ordered Random Variables from Exponentiated Pareto Distribution
}

\author{
Devendra Kumar* \\ Department of Statistics, Central University of Haryana, India
}

Submission: November 14, 2017; Published: May 22, 2018

*Corresponding author: Devendra Kumar, Department of Statistics, Central University of Haryana, India; Email: devendrastats@gmail.com

\begin{abstract}
In this paper, we have established several new explicit expressions and recurrence relations satisfied by the quotient moments and conditional quotient moments of dual generalized order statistics from Exponentiated Pareto distribution, to enable one to evaluate the single and product moments of all order in simple recursive manner. The results for order statistics and record values are deduced from the relations derived. Further, recurrence relation for conditional quotient moments of dual generalized order statistics we obtain a characterization of exponentiated Pareto distribution.

Keywords: Dual generalized order statistics; Order statistics; Record values; Exponentiated Pareto distribution; Quotient moments; Recurrence relations; Conditional expectation; Characterization; Statistics; Reliability theory; Random variables; Probability density; Relations quotient; Continuous distribution; Explicit expressions; Recurrence relations; Recursive manner; Dual generalized; Pfeifer records; Framework; Cumulative distribution
\end{abstract}

Abbreviations: PDF: Probability Density Function; CDF: Cumulative Distribution Function; EP: Exponentiated Pareto

\section{Introduction}

The concept of generalized order statistics (gos) was introduced by Kamps [1] as a general framework for models of ordered random variables. Moreover, many other models of ordered random variables, such as, order statistics, -th upper record values, upper record values, progressively Type II censoring order statistics, Pfeifer records and sequential order statistics are seen to be particular cases of These models can be effectively applied, e.g., in reliability theory. However, random variables that are decreasingly ordered cannot be integrated into this framework. Consequently, this model is inappropriate to study, e.g. reversed ordered order statistic and lower record values models. Burkschat et al. [2] introduced the concept of dual generalized order statistics (dgos). The dgos models enable us to study decreasingly ordered random variables like reversed order statistics, lower k-record values and lower Pfeirfer records, through a common approach below:

$$
\text { Suppose } Y_{d}(1, n, \tilde{m}, k), \ldots, \quad Y_{d}(n, n, \tilde{m}, k),
$$

$(k \geq 1, m$ is a real number), are $n d g o s$ from an absolutely continuous cumulative distribution function (cdf) $F(y)$ with probability density function (pdf) $f(y)$, if their joint pdf is of the form

$$
k\left(\prod_{j=1}^{n-1} \gamma_{j}\right)\left(\prod_{i=1}^{n-1}\left[F\left(y_{i}\right)\right]^{m_{i}} f\left(y_{i}\right)\right)\left[F\left(y_{i}\right)\right]^{k-1} f\left(y_{i}\right)
$$

for $F^{-1}(1)>y_{1} \geq y_{2} \geq \ldots \geq y_{n}>F^{-1}(0)$.

where $\gamma_{j}=k+(n-j)(m+1)>0$ for all $j, 1 \leq j \leq n, k$ is a positive integer and $m \geq-1$.

For convenience, let us define $Y(r, n, m, k)=0$. It can be seen that for $Y(r, n, m, k)=0$. i.e., $\gamma_{i}=n-i+1 ; 1 \leq i \leq n-1$, we obtain the joint pdf of the ordinary order statistics. In the similar manner, choosing the parameters appropriately, some other models such as $k^{\text {th }}$ lower record values $m_{r}=(n-i+1) \alpha_{i} ; 1 \leq i \leq n-1$, $\left(m_{1}=m_{2}=\cdots m_{n-1}=-1, k \in N\right.$, i.e., $\left.\gamma_{i}=k, 1 \leq i \leq n-1\right)$ sequential order statistic $\left(m_{r}=(n-r+1) \alpha_{r}-(n-r) \alpha_{r+1}-1 ; r=1, \ldots, n-1, k=\alpha_{n}\right.$; $\alpha_{1}, \alpha_{2}, \ldots, \alpha_{n}>0$, i. e. , $\left.\gamma_{i}=(n-i+1) \alpha_{i} ; 1 \leq i \leq n-1\right)$, order statistics with non-integral sample size $\left(m_{1}=\cdots=m_{n-1}=0\right.$, $k=\alpha-n+1$ with $n-1<\alpha \in R$, i.e., $\left.\gamma_{i}=\alpha-i+1 ; 1 \leq i \leq n-1\right)$ $[3,4]$, Pfeifer's record values $\left(m_{r}=\beta_{r}-\beta_{r+1}-1, r=1, \ldots, n-1\right.$ and $k=\beta_{n}$; $\beta_{1}, \beta_{2}, \ldots, \beta_{n}>0$, i.e., $\left.\gamma_{i}=\beta_{i} ; 1 \leq i \leq n-1\right)$ and progressively type-II right censored order statistics $\left(m_{i} \in N_{0}, k \in N\right)$ can be obtained [5].

In view of (1.1), the marginal pdf of $\mathrm{r}$-th $d g o s$ is given by

$$
\begin{aligned}
& f_{Y_{d}(r, n, m, k)}(x)=\frac{C_{r-1}}{(r-1) !}[F(y)]^{\gamma_{r-1}} f(y) g_{m}^{r-1}(F(y)) . \\
& f_{Y_{d}(r, n, m, k), Z_{d}(s, n, m, k)}(y, z)=\frac{C_{s-1}}{(r-1) !(s-r-1) !}[F(y)]^{m} f(y) g_{m}^{r-1}(F(y)) \\
& \times\left[h_{m}(F(z))-h_{m}(F(y))\right]^{s-r-1}[F(z)]^{\gamma_{s-1}} f(z), y>z,
\end{aligned}
$$


Where,

$$
\begin{gathered}
C_{r-1}=\prod_{i=1}^{r} \gamma_{i}, \\
h_{m}(y)=\left\{\begin{array}{cc}
-\frac{1}{m+1} y^{m+1}, & m \neq-1 \\
-\operatorname{In} y, & m=-1
\end{array}\right.
\end{gathered}
$$

and

$$
g_{m}(y)=h_{m}(y)-h_{m}(1), y \in[0,1) \text {. }
$$

Several authors like Pawlas \& Szynal [6], Ahsanullah [7], Mbah \& Ahsanullah [8], Khan \& Kumar [9,10], Kumar [11,12], Kumar [13] have done some work on dgos.
The remaining of the article is organized as follows. In Section 2, the cdf and pdf of exponentiated Pareto distribution are explained along with some graphs at some particular values of parameters. In Section 3, we derive the relations for quotient moments of exponentiated Pareto distribution. We obtained relations for conditional quotient moments of from this distribution in Section 4. In last section of the paper we prove a characterization result on this distribution based on recurrence relation for conditional quotient moment of the dgos.

\section{The exponentiated pareto distribution}
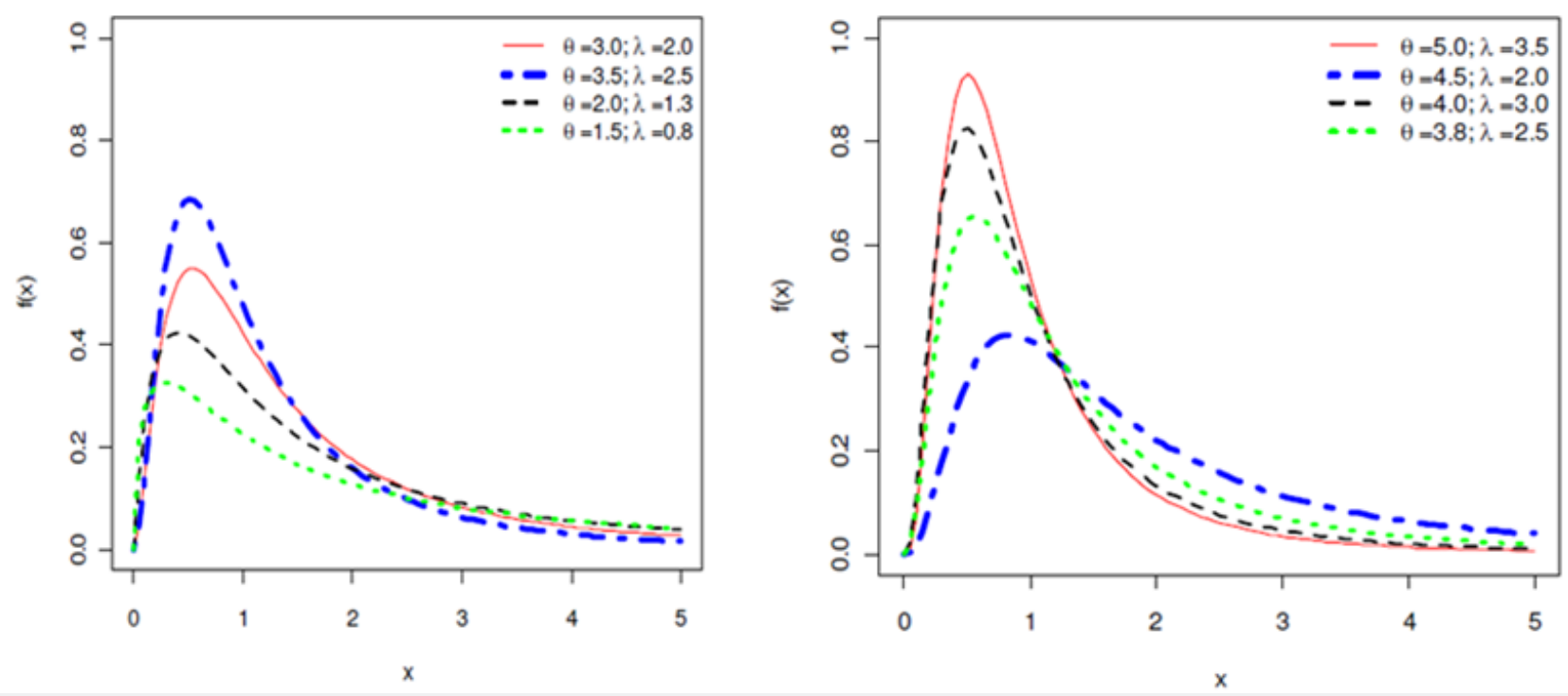

Figure 1: Plots of the EP distribution pdf for some parameter values.
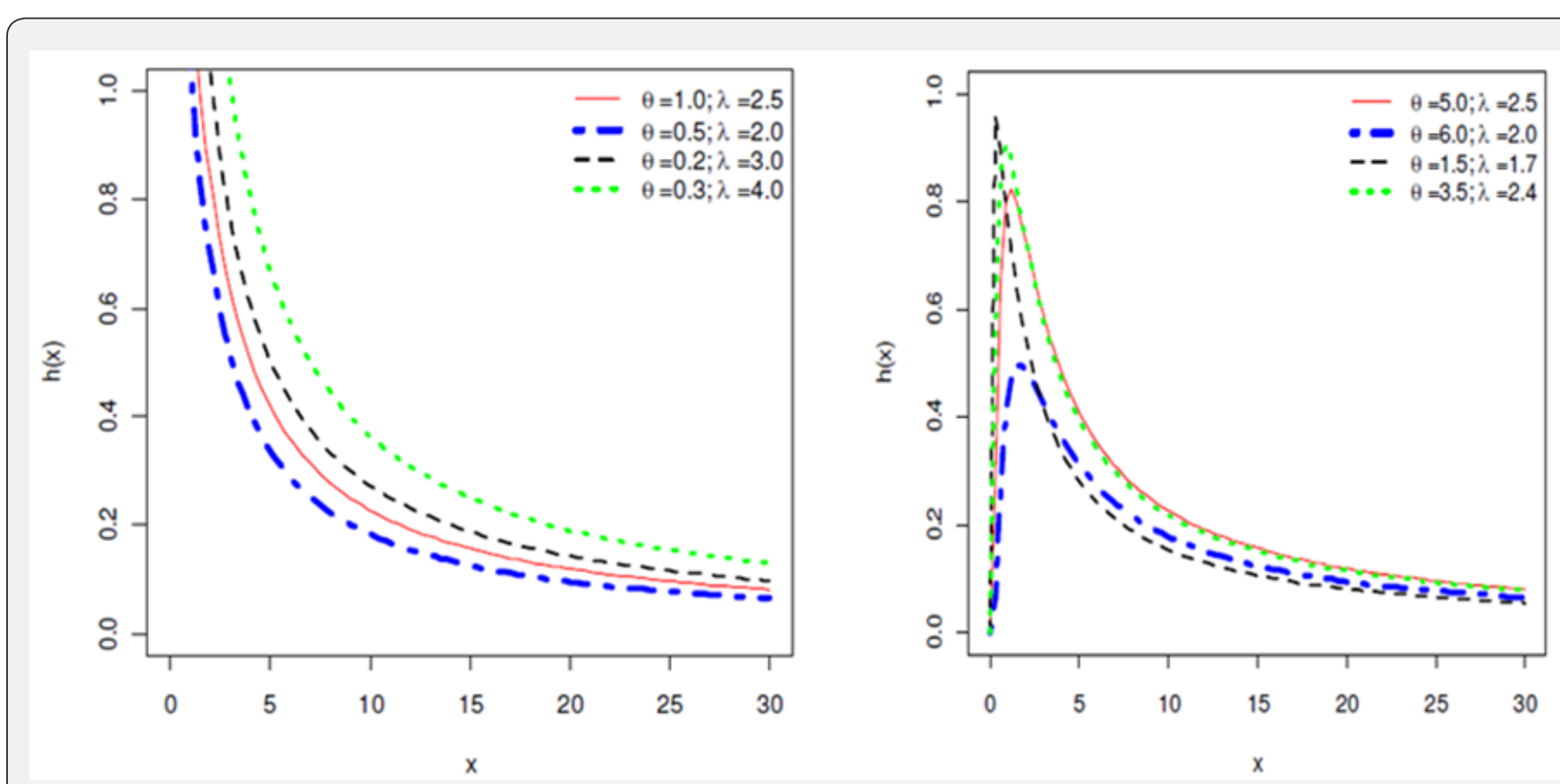

Figure 2: Plots of the EP distribution hazard function for some parameter values. 

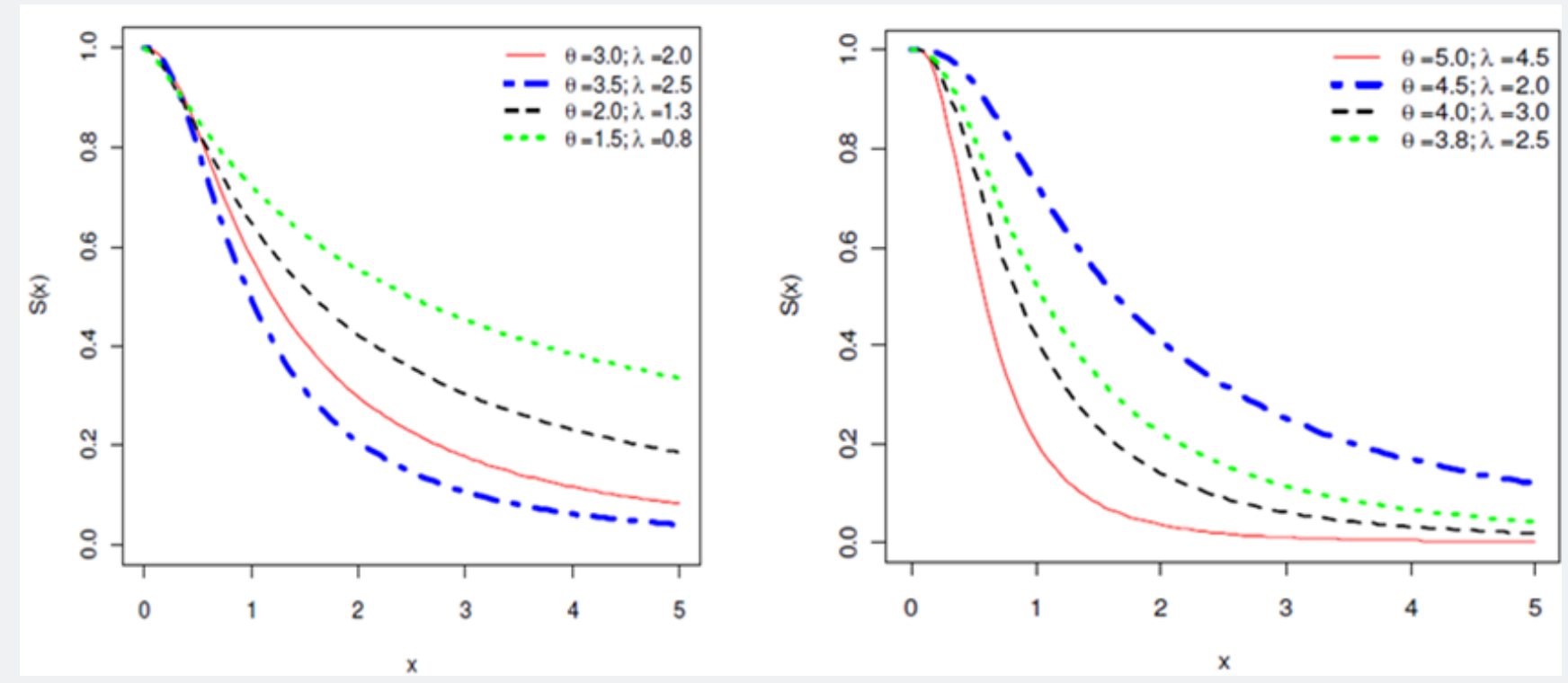

Figure 3: Plots of the EP distribution survival function for some parameter values.

The exponentiated Pareto (EP) distribution was first introduced in the literature by Gupta et al. [14]. The probability density function (pdf) is given by

$$
f(y ; \alpha, \theta)=\theta \lambda\left[1-(1+y)^{-\lambda}\right]^{\theta-1}(1+y)^{-(\lambda+1)}, \quad y>0, \lambda, \theta>0
$$

and the cumulative distribution function (cdf) is

$$
F(y ; \alpha, \theta)=\left[1-(1+y)^{-\lambda}\right]^{\theta}, y>0, \lambda, \theta>0,
$$

the survival function

$$
S(y ; \varepsilon, \theta)=1-\left[1-(1+y)^{-\lambda}\right]^{\theta}, y>0, \lambda, \theta>0
$$

and the hazard function

$$
h(y ; \alpha, \beta)=\frac{\theta \lambda\left[1-(1+y)^{-\lambda}\right]^{\theta-1}(1+y)^{-(\lambda+1)}}{1-\left[1-(1+y)^{-\lambda}\right]^{\theta}} .
$$

One can observe from equation (1.1) and (1.2) that the characterizing differential equation for EP distribution is given by

$$
F(y ; \alpha, \theta)=\frac{1}{\theta \lambda}\left[\lambda y+\sum_{t=2}^{\lambda+1}\left(\begin{array}{c}
\lambda+1 \\
t
\end{array}\right) y^{t}\right] f(y ; \alpha, \beta), \lambda
$$

Where $\theta$ and $\lambda$ are two shape parameters. When $\theta=1$ the above distribution corresponds to the standard Pareto distribution of second kind [15] (Figure 1-3).

\section{Relations for quotient moments of dgos}

Lemma 3.1: For distribution as given in (2.1) and non-negative integers $\alpha, \beta, \tau$

$$
\begin{aligned}
& \phi_{i, j}(\alpha, 0, \tau)=\theta^{2} \sum_{p=0}^{\infty} \sum_{q=0}^{\infty} \sum_{l=0}^{\infty} \sum_{w=0}^{i}(-1)^{i-j-w-1}\left(\begin{array}{c}
i \\
w
\end{array}\right) \frac{(j+1)_{(p)}(p / \lambda)_{(q)}(w / \lambda)_{(l)}}{p ! q ! l ![\theta[\tau+1]+q]} \\
& \times \frac{1}{[\theta(\alpha+\tau+2)+l+q]},
\end{aligned}
$$

Where,

$$
\phi_{i, j}(\alpha, \beta, \tau)=\int_{0}^{\infty} \int_{0}^{y} \frac{y^{i}}{z^{j+1}}[F(y)]^{\alpha} f(y)\left[h_{m}(F(z))-h_{m}(F(y))\right]^{\beta}[F(z)]^{\tau} f(z) d z d y .
$$

Proof: From (3.2), we have

$$
\phi_{i . j}(\alpha, 0, \tau)=\int_{0}^{\infty} y^{i}[F(y)]^{\alpha} f(y) P(y) d y,
$$

Where,

$$
P(y)=\int_{0}^{y} z^{-(j+1)}[F(z)]^{\tau} f(z) d z .
$$

By setting in (3.4) and simplifying the resultant expression, we obtain the relation in (3.1).

Lemma 3.2: For the distribution as given in (2.1) and any nonnegative integers and

$$
\begin{aligned}
& \phi_{i, j}(\alpha, \beta, \tau)=\frac{\theta^{2}}{(m+1)^{\beta}} \sum_{p=0}^{\infty} \sum_{q=0}^{\infty} \sum_{l=0}^{\infty} \sum_{v=0}^{\beta} \sum_{w=0}^{i}(-1)^{i+v-w-j-1}\left(\begin{array}{c}
i \\
w
\end{array}\right) \frac{(j+1)_{(p)}(p / \lambda)_{(q)}}{p ! q ! l !} \\
& \times \frac{(w / \lambda)_{(1)}}{[\theta\{\tau+1+v(m+1)\}+q][\theta\{\alpha+\tau+2+\beta(m+1)\}+p+l]}, m \neq-1 \\
& =\beta ! \theta^{\beta+2} \sum_{p=0}^{\infty} \sum_{q=0}^{\infty} \sum_{l=0}^{\infty} \sum_{w=0}^{i}(-1)^{i-w-j-1}\left(\begin{array}{c}
i \\
w
\end{array}\right) \frac{(j+1)_{(p)}(p / \lambda)_{(q)}(w / \lambda)_{(l)}}{p ! q ! l !} \\
& \times \frac{1}{[\theta(\tau+1)+q]^{\beta+1}[\theta(\alpha+\tau+2)+p+l]}, m=-1 .
\end{aligned}
$$
(3.5).

When $m=-1$, we have

$\phi_{i, j}(\alpha, \beta, \tau)=\frac{0}{0}$ as $\sum_{v=0}^{\beta}(-1)^{v}\left(\begin{array}{l}\beta \\ v\end{array}\right)=0$.

Since (3.5) is of the form $\frac{0}{0}$ at $m=-1$, therefore, we have

$\phi_{i, j}(\alpha, \beta, \tau)=\psi \sum_{v=0}^{\beta}(-1)^{v}\left(\begin{array}{l}\beta \\ v\end{array}\right) \frac{[\theta\{\tau+1+v(m+1)\}+q]^{-1}}{(m+1)^{b}[\theta\{\alpha+\tau+2+\beta(m+1)+p+1\}]}$,

Where,

$$
\psi=\theta^{2} \sum_{p=0}^{\infty} \sum_{q=0}^{\infty} \sum_{l=0}^{\infty} \sum_{w=0}^{i}(-1)^{i-w-j-1}\left(\begin{array}{c}
i \\
w
\end{array}\right) \frac{(j+1)_{(p)}(p / \lambda)_{(q)}(w / \lambda)_{(l)}}{p ! q ! l !} .
$$


Differentiating of (3.7) $\beta$ times with respect to $m$, we get

$$
\phi_{i, j}(\alpha, \beta, \tau)=\psi \theta^{\beta} \sum_{v=0}^{\beta}(-1)^{v+\beta}\left(\begin{array}{l}
\beta \\
v
\end{array}\right) \frac{v^{\beta}[\theta(\tau+1)+q]^{-(\beta+1)}}{[\theta(\alpha+\tau+2)+p+l]}, \beta>0 .
$$

On applying L' Hospital rule, we have

$\lim _{m \rightarrow-1} \phi_{i, j}(\alpha, \beta, \tau)=\psi \theta^{\beta} \sum_{v=0}^{\beta}(-1)^{v+\beta}\left(\begin{array}{l}\beta \\ v\end{array}\right) \frac{v^{\beta}[\theta(\tau+1)+q]^{-(\beta+1)}}{[\theta(\alpha+\tau+2)+p+l]}$.

Hence we have the result, [16] given in (3.6).

Theorem 3.1: For $1 \leq r \leq s-2, k=1,2, \ldots, i=1,2, \ldots, j=1,2, \ldots$,

$$
\begin{aligned}
& E\left[\frac{Y_{d}^{i}(r, n, m, k)}{Y_{d}^{j+1}(s, n, m, k)}\right]=\frac{\theta^{2} C_{s-1}}{(r-1) !(s-r-1) !(m+1)^{s-2}} \sum_{p=0}^{\infty} \sum_{q=0}^{\infty} \sum_{l=0}^{\infty} \sum_{u=0}^{r-1} \sum_{v=0}^{s-r-1} \sum_{w=0}^{i}(-1)^{i-u+v-j-w-1} \\
& \times\left(\begin{array}{c}
r-1 \\
u
\end{array}\right)\left(\begin{array}{c}
s-r-1 \\
v
\end{array}\right)\left(\begin{array}{c}
i \\
w
\end{array}\right) \frac{(j+1)_{(p)}(p / \lambda)_{(q)}(w / \lambda)_{(l)}}{p ! q ! l !\left[\theta \gamma_{s-v}+q\right]\left[\theta \gamma_{r-u}+p+1\right]} .
\end{aligned}
$$

Proof The proof can be established on the lines of Lemma 3.2.

Remark 3.1: Under the assumption of Theorem 3.1 with $m=0, k=1$ we shall deduced the relations quotient moments of ordinary order statistics of the EP distribution.

Remark 3.2: Putting $k=0, m=-1$ in Theorem 3.1 we obtain the relations for quotient moments of lower record values for EP distribution.

Theorem 3.2: For $1 \leq r \leq s-2, k \geq 1, i=0,1,2, \ldots$, and $j=1,2, \ldots$, $\left(1-\frac{j+1}{\theta \lambda_{s}}\right) E\left[\frac{Y_{d}^{i}(r, n, m, k)}{Y_{d}^{j+1}(s, n, m \cdot k)}\right]=E\left[\frac{Y_{d}^{i}(r, n, m, k)}{Y_{d}^{j+1}(s-1, n, m \cdot k)}\right]$ $+\frac{(j+1)}{\theta \lambda \gamma_{s}} \sum_{t=0}^{\lambda+1}\left(\begin{array}{c}\lambda+1 \\ t\end{array}\right) E\left[\frac{Y_{d}^{i}(r, n, m, k)}{Y_{d}^{j-t+1}(s, n, m \cdot k)}\right]$

Proof: For $1 \leq r \leq s-2, k \geq 1, i=0,1,2, \ldots$, and $j=1,2, \ldots$, we have from (1.2) and (1.3),

$E\left[\frac{Y_{d}^{i}(r, n, m, k)}{Y_{d}^{j+1}(s, n, m . k)}\right]=\frac{C_{s-1}}{(r-1) !(s-r-1) !} \int_{0}^{\infty} Y^{i}\left[F(y)^{m}\right] f(y) g_{m}^{r-1}(F(y)) L(y) d y$

Where,

$$
L(y) \int_{0}^{y} \frac{1}{z^{j+1}}\left[h_{m}(F(z))-h_{m}(F(y))\right]^{s-r-1}[F(z)]^{\gamma_{s}-1} f(z) d z .
$$

Integrating $L(y)$ by parts and using (2.4) and substituting the resultant expression in (3.11), we get the result given in (3.10).

Remark 3.3: Under the assumptions of Theorem 3.2, with $m=0, k=1$ we shall deduce the recurrence relations for quotient moments of ordinary order statistics from the EP distribution.

Remark 3.4: Putting $k=0, m=-1$ in theorem 3.2, we obtain the recurrence relations for quotient moments of lower record of the EP distribution.

\section{Relations for quotient conditional expectation}

Theorem 4.1: For the distribution given in (2.1) and for $1 \leq r \leq s \leq n-2, i=0,1,2, \ldots, j=1,2, \ldots$, and $k=1,2, \ldots$,

$$
\begin{aligned}
& E=\left[\frac{Y_{d}^{i}(r, n, m, k)}{Y_{d}^{j}(s, n, m \cdot k)} \mid Y_{d}(r, n, m, k)=y\right]=y^{i} \sum_{p=0}^{\infty} \sum_{q=0}^{\infty}(-1)^{-j} \frac{(j)_{(p)}(p / \lambda)_{(q)}}{p ! q !} \\
& \times\left[1-(1+y)^{-\lambda}\right]^{p} \prod_{v=0}^{s-l}\left(\frac{\gamma_{l+v}}{\gamma_{l+v}+q / \theta}\right), \quad l=r, r+1 .
\end{aligned}
$$

Proof: For $1 \leq r \leq s-2, i=1,2, \ldots, j=1,2, \ldots$, we have from (1.2), and (1.3)

$$
\begin{aligned}
& E=\left[\frac{Y_{d}^{i}(r, n, m, k)}{Y_{d}^{j}(s, n, m \cdot k)} \mid Y_{d}(r, n, m, k)=y\right]=\frac{y^{i} C_{s-1}}{(s-r-1) ! C_{r-1}(m+1)^{s-r-1}} \\
& \times \int_{0}^{y} y^{-j}\left[1-\left(\frac{F(z)}{F(y)}\right)^{m+1}\right]^{s-r-1}\left(\frac{F(z)}{F(y)}\right)^{\gamma_{s}-1} \frac{f(z)}{f(y)} d z .
\end{aligned}
$$

By setting $t=\frac{F(z)}{F(y)}$ from (2.2) in (3.2), we obtain

$$
\begin{aligned}
& E=\left[\frac{Y_{d}^{i}(r, n, m, k)}{Y_{d}^{j}(s, n, m \cdot k)} \mid Y_{d}(r, n, m, k)=y\right]=\frac{y^{i} C_{s-1}(-1)^{-j}}{(s-r-1) ! C_{r-1}(m+1)^{s-r-1}} \\
& \times \int_{0}^{y} y^{-j}\left[1-\left\{1-\left[1-(1+y)^{-\lambda}\right] t^{1 / \theta}\right\}^{-1 / \lambda}\right]^{-j} t^{\gamma_{s-1}}\left(1-t^{m+1}\right)^{s-r-1} d t
\end{aligned}
$$

$=\frac{y^{i} C_{s-1}(-1)^{-j}}{(s-r-1) ! C_{r-1}(m+1)^{s-r-1}} \sum_{p=0}^{\infty} \sum_{q=0}^{\infty} \frac{(j)_{(p)}(p / \lambda)_{(q)}\left[1-(1+y)^{-\lambda}\right]^{q}}{p ! q !}$

$\times \int_{0}^{y} t^{\gamma_{s}+(q / \theta)-1}\left(1-t^{m+1}\right)^{s-r-1} d t$

Again by setting $w=t^{m+1}$ in (4.3) and simplifying the resultant expression, we get relation in (4.1).

Remark 4.1: Under the assumptions of Theorem 4.1, with $m=0, k=1$ we shall deduce the relations for conditional quotient moments of ordinary order statistics from the EP distribution.

Remark 4.2: Putting $k=0, m=-1$ in theorem 4.1, we obtain the relations for conditional quotient moment of lower record of the EP Pareto distribution.

Theorem 4.2: For $1 \leq r \leq s-2, k \geq 1, i=0,1,2, \ldots$, and $j=1,2, \ldots$,

$$
\begin{aligned}
& \left(1-\frac{j+1}{\theta \gamma_{s}}\right) E\left[\frac{Y_{d}^{i}(r, n, m, k)}{Y_{d}^{j+1}(s, n, m \cdot k)} \mid Y_{d}(r, n, m, k)=x\right]=E\left[\frac{Y_{d}^{i}(r, n, m, k)}{Y_{d}^{j+1}(s-1, n, m \cdot k)} \mid Y_{d}(r, n, m, k)=x\right] \\
& +\frac{j+1}{\theta \gamma_{s}} \sum_{t=0}^{\lambda+1}\left(\begin{array}{c}
\lambda+1 \\
t
\end{array}\right) E\left[\frac{Y_{d}^{i}(r, n, m, k)}{Y_{d}^{j-t+1}(s, n, m \cdot k)} \mid Y_{d}(r, n, m, k)=x\right] .
\end{aligned}
$$

Proof: For $1 \leq r \leq s-2, k \geq 1, i=0,1,2, \ldots$, and $j=1,2, \ldots$, we have from (1.2) and (1.3), we have

$E\left[\frac{Y_{d}^{i}(r, n, m, k)}{Y_{d}^{j+1}(s, n, m . k)} \mid Y_{d}(r, n, m, k)=x\right]=\frac{y^{i} C_{s-1} L(y)}{(s-r-1) ! C_{r-1}[F(y)]^{\gamma_{r+1}}}$

Substituting the value of $L(y)$ from (3.12) in (4.5) and simplifying the resultant expression we get the relation in (4.4).

Remark 4.3: Under the assumptions of Theorem 4.2, with $m=0, k=1$ we shall deduced the recurrence relations for conditional quotient moments of ordinary order statistics from the EP distribution.

Remark 4.4: Putting $k=0, m=-1$ in theorem 4.2, we obtain the recurrence relations for conditional quotient moments of 
lower record of the EP Pareto distribution.

\section{Characterization}

Theorem 5.1: Let $Y$ be a non-negative random variable having an absolutely continuous distribution function $F(y)$ with $F(0)=0$ and $0<F(y)<1$ for all $x>0$, then

$$
\begin{aligned}
& \left(1-\frac{j+1}{\theta \gamma_{s}}\right) E\left[\frac{Y_{d}^{i}(r, n, m, k)}{Y_{d}^{j+1}(s, n, m \cdot k)} \mid Y_{d}(r, n, m, k)=y\right] \\
& =E\left[\frac{Y_{d}^{i}(r, n, m, k)}{Y_{d}^{j+1}(s-1, n, m \cdot k)} \mid Y_{d}(r, n, m, k)=y\right] \\
& +\frac{j+1}{\theta \gamma_{s}} \sum_{t=0}^{\lambda+1}\left(\begin{array}{c}
\lambda+1 \\
t
\end{array}\right) E\left[\frac{Y_{d}^{i}(r, n, m, k)}{Y_{d}^{j-t+1}(s, n, m \cdot k)} \mid Y_{d}(r, n, m, k)=x\right] .
\end{aligned}
$$

if and only if

$$
F(z)=\left[1-(1+z)^{-\lambda}\right]^{\theta}, z>0, \theta>0 .
$$

Proof: From (1.2) and (1.3) and using (5.1) we have

$$
\frac{C_{s-1}}{C_{r-1}(s-r-1) !} \int_{0}^{y} \frac{y^{i}}{z^{j+1}}\left[h_{m}(F(z))-h_{m}(F(y))\right]^{s-r-2} \frac{[F(z)]^{\gamma_{s-1}-1}}{[F(z)]^{\gamma_{r+1}}} f(z) d z
$$$$
=\frac{C_{s-2}}{C_{r-1}(s-r-2) !} \int_{0}^{y} \frac{y^{i}}{z^{j+1}}\left[h_{m}(F(z))-h_{m}(F(y))\right]^{s-r-2} \frac{[F(z)]^{\gamma_{s-1}-1}}{[F(z)]^{\gamma_{r+1}}} f(z) d z
$$$$
+\frac{(j+1) C_{s-1}}{\theta \gamma_{s} C_{r-1}(s-r-2) !} \int_{0}^{y} \frac{y^{i}}{z^{j+1}}\left[h_{m}(F(z))-h_{m}(F(y))\right]^{s-r-1} \frac{[F(z)]^{\gamma_{s-1-1}}}{[F(z)]^{\gamma_{r+1}}} f(z) d z
$$$$
+\frac{(j+1) C_{s-1}}{\lambda \theta \gamma_{s} C_{r-1}(s-r-1) !} \sum_{t=2}^{\lambda+1}\left(\begin{array}{c}
\lambda+1 \\
t
\end{array}\right) \int_{0}^{y} \frac{y^{i}}{z^{j+1}}\left[h_{m}(F(z))-h_{m}(F(y))\right]^{s-r-1} \times \frac{[F(z)]^{\gamma_{s}-1}}{[F(y)]^{\gamma_{r+1}}} f(z) d z
$$

Integrating (4.2) by parts, we get

$$
\begin{aligned}
& \frac{(j+1) C_{s-1}}{\gamma_{s} C_{r-1}(s-r-1) !} \int_{0}^{y} \frac{y^{i}}{z^{j+2}}\left[h_{m}(F(z))-h_{m}(F(y))\right]^{s-r-1} \frac{[F(z)]^{\gamma_{s-1}}}{[F(y)]^{\gamma_{r+1}}} \\
& \times\left\{F(z)-\frac{z}{\theta} f(z)-\frac{1}{\theta \lambda} \sum_{t=2}^{\lambda+1}\left(\begin{array}{c}
\lambda+1 \\
t
\end{array}\right) z^{t} f(z)\right\} d z=0 .
\end{aligned}
$$

From Müntz-Szász Theorem to equation (5.3) [17], we get

$$
\frac{f(z)}{F(z)}=\left[\frac{z}{\theta}+\frac{1}{\theta \lambda} \sum_{t=2}^{\lambda+1}\left(\begin{array}{c}
\lambda+1 \\
t
\end{array}\right) z^{t}\right]^{-1}
$$

which proves that

$$
\frac{f(z)}{F(z)}=\left[\frac{z}{\theta}+\frac{1}{\theta \lambda} \sum_{t=2}^{\lambda+1}\left(\begin{array}{c}
\lambda+1 \\
t
\end{array}\right) z^{t}\right]^{-1} z>0, \lambda, \theta>0 .
$$

\section{References}

1. Kamps U (1995) A Concept of Generalized Order Statistics, B.G. Teubner Stuttgart.

2. Burkschat M, Cramer E, Kamps U (2003) Dual generalized order statistics. Metron 9(1): 13-26.

3. Rohatgi VK, Saleh AK (1988) A class of distributions connected to order statistics with non integral sample size. Commun Statist Theory Meth 17(6): 2005-2012.

4. Saleh AK, Scott C, Junkins DB (1975) Exact first and second order moments of order statistics from truncated exponential distribution. Naval Research Logistics Quarterly 22: 65-77.

5. Kamps U, Cramer E (2001) On distributions of generalized order statistics. Statistics 35: 269-280.

6. Pawlas P, Szynal D (2001) Recurrence relations for single and product moments of lower generalized order statistics from the inverse Weibull distribution. Demonstratio Math 34: 353-358.

7. Ahsanullah M (2004) A characterization of the uniform distribution by dual generalized order statistics, Comm Statist Theory and Methods 33: 2921-2928.

8. Mbah AK, Ahsanullah M (2007) Some characterization of the power function distribution based on lower generalized order statistics. Pakistan Journal of Statistics 23: 139-146.

9. Khan RU, Kumar D (2011) Lower generalized order statistics from exponentiated gamma distribution and its characterization. ProbStats Forum 4: 25-38.

10. Khan RU, Kumar D (2011) Expectation identities of lower generalized order statistics from generalized exponential distribution and a characterization. Math. Methods Statist 20: 150-157.

11. Kumar D (2011) On relations for generalized Rayleigh distribution based on lower generalized order statistics and its characterization. Journal of Statistical Research 45: 49-57.

12. Kumar D (2011) Explicit expressions for moments of lower generalized order statistics from exponentiated Kumaraswamy distribution and its characterization. Journal of Applied Probability and Statistics 6: 61-72.

13. Kumar D (2012) Relations for moments of lower generalized order statistics from a family of J-shaped distributions and its characterization. Journal of Applied Probability and Statistics 7: 71-86.

14. Gupta RC, Gupta RD, Gupta PL (1998) Modeling failure time data by Lehman alternatives. Comm Statist Theory Methods 27: 887-904.

15. Johnson NL, Kotz S, Balakrishnan N (1994) Continuous Univariate Distributions. John Wiley New York, USA.

16. Ruiz SM (1996) An algebraic identity leading to Wilson's theorem. Math Gaz 80: 579-582.

17. Hwang JS, Lin GD (1984) On a generalized moments problem II. Proc Amer Math Soc 91: 577-580. 


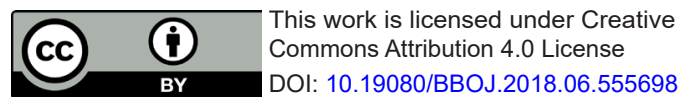

\section{Your next submission with Juniper Publishers will reach you the below assets}

- Quality Editorial service

- Swift Peer Review

- Reprints availability

- E-prints Service

- Manuscript Podcast for convenient understanding

- Global attainment for your research

- Manuscript accessibility in different formats

( Pdf, E-pub, Full Text, Audio)

- Unceasing customer service

Track the below URL for one-step submission https://juniperpublishers.com/online-submission.php 